\title{
The Robotic Water Polo and Underwater Robot Cooperation Involved in the Game
}

\author{
Zhang Lee, Guangming Xie, Dandan Zhang and Jinyan Shao \\ Intelligent Control Laboratory, College of Engineering, Peking University
}

China

\section{Introduction}

Multi-robot cooperation is a very important area in the field of robot. As we know, it is difficult to use a single robot to complete tasks independently. Often, people use multiple robots cooperation in order to compensate for the lack of individual ability. Multi-level robot can greatly improve the efficiency of the whole system. Compared to a single robot, a coordinated multi-robot system has many advantages : First, because of multi-robot systems' distribution in space, resources and functions, multi-robot systems have higher efficiency and wider scope of the application than single robot; Second, because multi-robot systems have high redundancy, So multi-robot systems have more fault-tolerant capabilities and higher robust than single robot; Also compared with the design of a very powerful independent robot, construction of a number of robots which have simple structure and function will be economical, easy, flexible, and can greatly reduce the costs. Due to the

$E$ advantages of the multi-robot systems, Multi-robot cooperation is drawing increasing $\delta$ attention to the people, a lot of researches on multi-robot coordination and centralization, $\stackrel{ \pm}{\subseteq}$ Load distribution, motion planning and other issues have been done all over the world.

By now, multi-robot cooperation achievements were mainly concentrated in the areas of 응 robots on land, there has few achievements of underwater robot collaboration. The main \& reason is that the complexity and uncertainty of the underwater environment bring a lot of interference to the system, the efficiency and accuracy of the control are reduced to a large extent by these interfere. In addition, the underwater environment has a higher requirement 3 that the robot should have a higher compressive strength, anti-jamming capabilities and \& more accurate and robust sensing system. In fact, along with the exploration of underwater 웜 resources, there are more and more underwater tasks, many underwater tasks need a సँ number of robots to collaborate because of their high complexity. The research on is underwater robot collaboration has become an urgent task.

\& We propose a novel robotic soccer game called Robotic Water Polo(RWP) to promote the O underwater robot technique and their combination, Fig.1 shows the filed of RWP, Similar to the popular robotic soccer games on land, the RWP is also designed as standard task for Фे multiple swimming robots under a dynamic underwater environment. To play RWP, each O team has three or two underwater robots, we called them robotic fish. They do their best to

Source: Robotic Soccer, Book edited by: Pedro Lima, ISBN 978-3-902613-21-9, pp. 598, December 2007, Itech Education and Publishing, Vienna, Austria 
push the ball to their opponent's gate, Fig.1(b) and (c)display the procedure of the competition. Because the special environment of the game, the RWP also involves hydrodynamic analysis, underwater communications, underwater image processing, anti-jamming technology, and other aspects of technology which is more difficult, more complicated than the similar game on land.

We focus this chapter on introducing the underwater robot cooperation involved in RWP and the basis of RWP, due to space limitation, we can't elaborate on all the cooperative technologies involved in RWP. We only introduce collision avoidance of robotic fish and the multiple robot fish cooperation system of we used in RWP now, in section 2, we first introduce the prototype of the underwater robot called robotic fish, in section 3 we introduce a general task-oriented platform which provides both hardware and software foundations for cooperation of underwater robots, in section 4 we propose a new reactive collision avoidance method for robotic fish navigation in RWP, in section 5 a situation based action selection mechanism is proposed for pushing ball to goal in RWP, and finally in section 6 , there will be a conclusion.

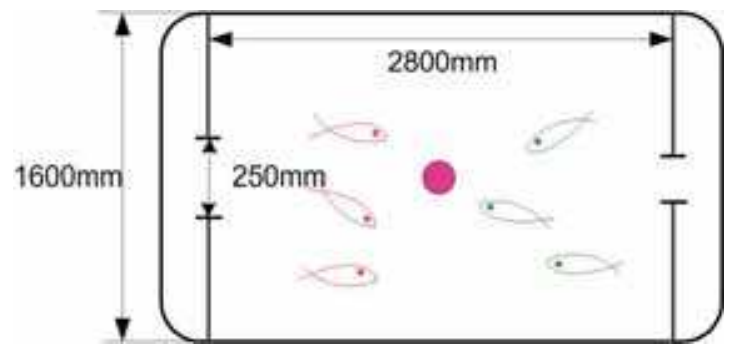

(a) Field of RWP(Used by Peking university)

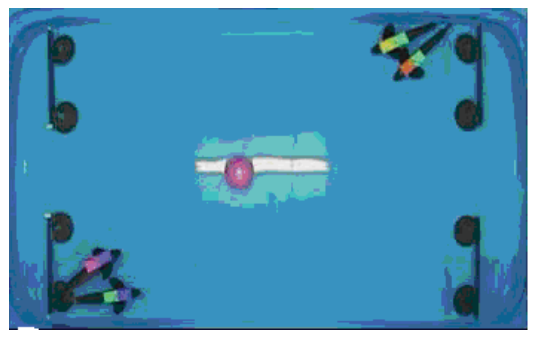

(b)Start of RWP

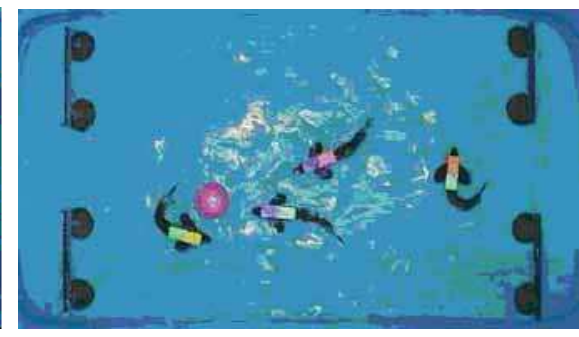

(c) Fragment of RWP

Fig.1. Field and display of RWP

\section{The Prototype of Robotic Fish}

Before introducing the underwater robot cooperating technologies, we first present the robotic fish prototype developed for RWP in our laboratory, because it is the basis of RWP. Fig 2 shows the prototype of robot fish: 


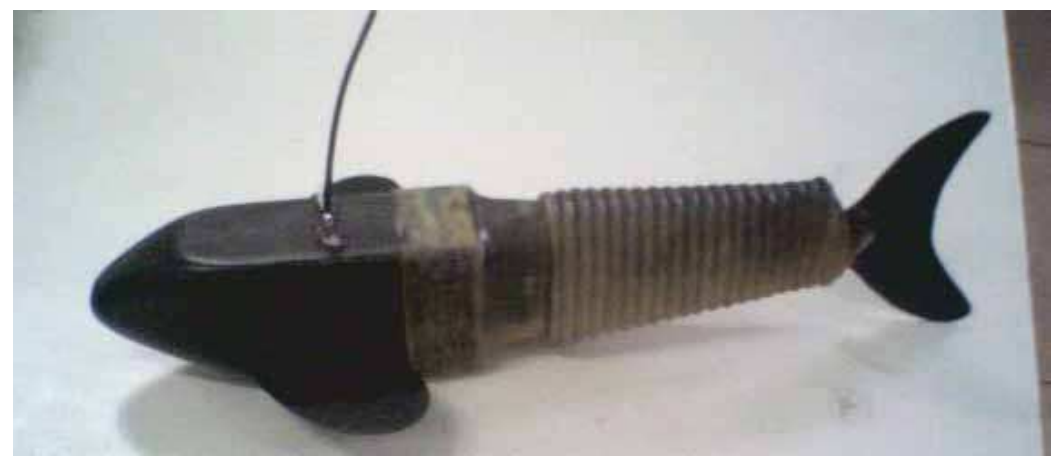

Fig. 2. Prototype of robot fish used in RWP

This multiple-mode robot-fish is mainly used to realize the cooperative control of multiple robots underwater. And the advantages of the fish are low cost, small size, and good agility, stability and accuracy. In the environment of lab or competition, the location information of the robot-fish can be gotten from the global vision, so we can conveniently control the cooperative movements of the multiple-robot fish.

The structure of robot-fish can mainly be divided into three parts: fish head, body, tail.The fish head portion is composed of the control circuit board, battery, communication module, power switch and pectoral fin.The fish body portion is composed of three in series swaying joints, and each joint is driven by the direct current servo motor, in order to simulate the wave curvilinear motion of the fish's body. The fish tail is tail fin of the robot-fish. The mechanical structure of the robot-fish is as the Fig 2, For technical details on design and implementation of the robotic fish. We can't elaborate on them here due to space limitation.
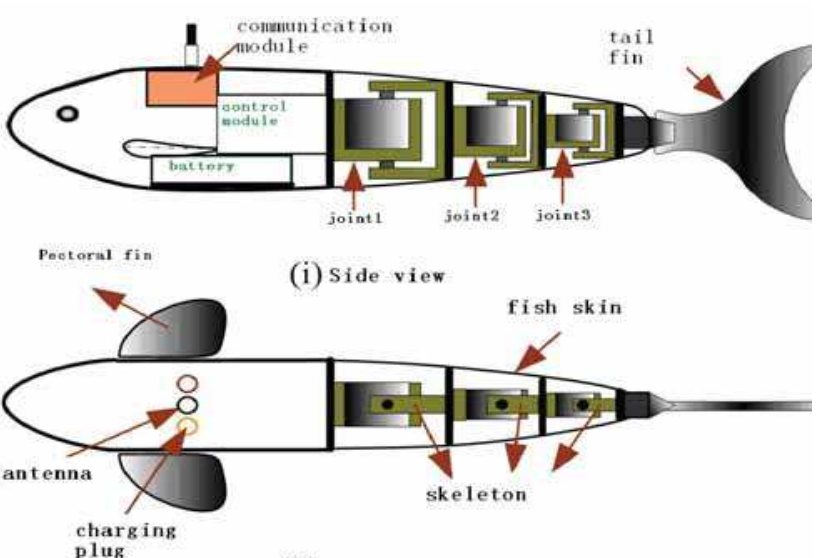

(ii) Vertical view

Fig. 3. The mechanical structure of the robot-fish 
This type of multiple-mode robot-fish involved in the lab presently can complete precision control in the mission of multiple-robot-fish cooperative control, and also can ensure both the stability of the robot-fish's swimming and accuracy of the swimming performance. We have tested the robot-fish repeatedly through experimentation. It can satisfy the requirement of the RWP and other cooperative control.

\section{The Multiple Robotic Fish Cooperation System}

In recent years, underwater biomimetic robotics has emerged as a challenging new research topic, which combines bioscience engineering technology and underwater robotics, aiming at developing new classes of robots which will be substantially more compliant and stable than current robots. Taking advantage of new developments in materials, fabrication technologies, sensors and actuators, more and more biologically inspired robots have been developed. As one of the hot topics, robotic fish has received considerable attention during the last decade. Fish, after a long history's natural selection, have evolved to become the best swimmers in nature. They can achieve tremendous propulsive efficiency and excellent maneuverability with little loss of stability by coordinating their bodies, fins and tails properly. Researchers believe that these remarkable abilities of fish can inspire innovative designs to improve the performance, especially maneuverability and stabilization of underwater robots.

By now, the research on robotic fish mainly focuses on design and analysis on individual robot fish prototype, while seldom is concerned with the cooperation behaviors of the fish. As we know, fish in nature often swim in schools to strive against the atrocious circumstances in the sea. Similarly, in practice, the capability of a single robot fish is limited and it will be incompetent for achieving complex missions in dynamic environments. Thus, for real-world (ocean based) applications, a cooperative multiple robot fish system is required, which is the motivation of this work.

In this section, we propose the development of the Multiple Robot Fish cooperation System (MRFS), which is built on the basis of a series of radio-controlled, multi-link biomimetic robot fish. There are two features in MRFS: first, this cooperative platform is general and can be applied to different types of cooperative tasks; second, high-level tasks are eventually decomposed into two reactive motion controllers, which are designed under full consideration on the inertia of fish and the hydrodynamic forces of surrounding water.

The remaining of the section is organized as follows. In subsection 3.1 we present the establishment of the platform for MRFS. Based on the hardware and software platform, a four-level hierarchical control architecture is provided in subsection 3.2.

\subsection{Design and Implementation of MRFS Platform}

As mentioned above, a single fish is often limited both in capabilities and movement range. It will be incompetent for many complex tasks in dynamic environments. In this case, a multi-robot fish cooperative system becomes a desired solution. Inspired by the technology of multi-agent system and the approaches developed for cooperation of ground mobile robots, we establish the hardware platform of MRFS as depicted in Fig 4. The whole system can be decomposed into four subsystems: robot fish subsystem, image capturing subsystem, 
decision making and control subsystem and wireless communication subsystem.
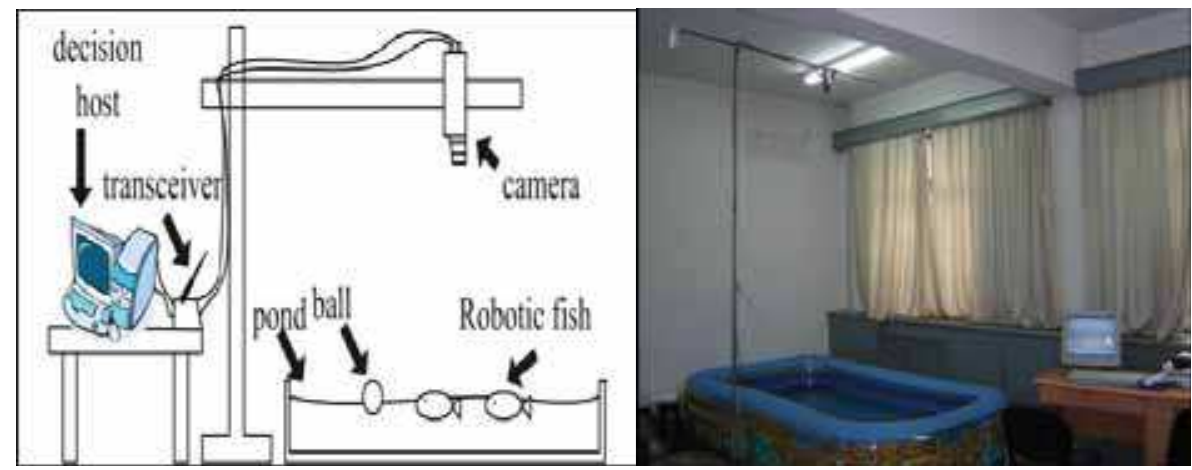

Fig. 4. Hardware platform for MRFS

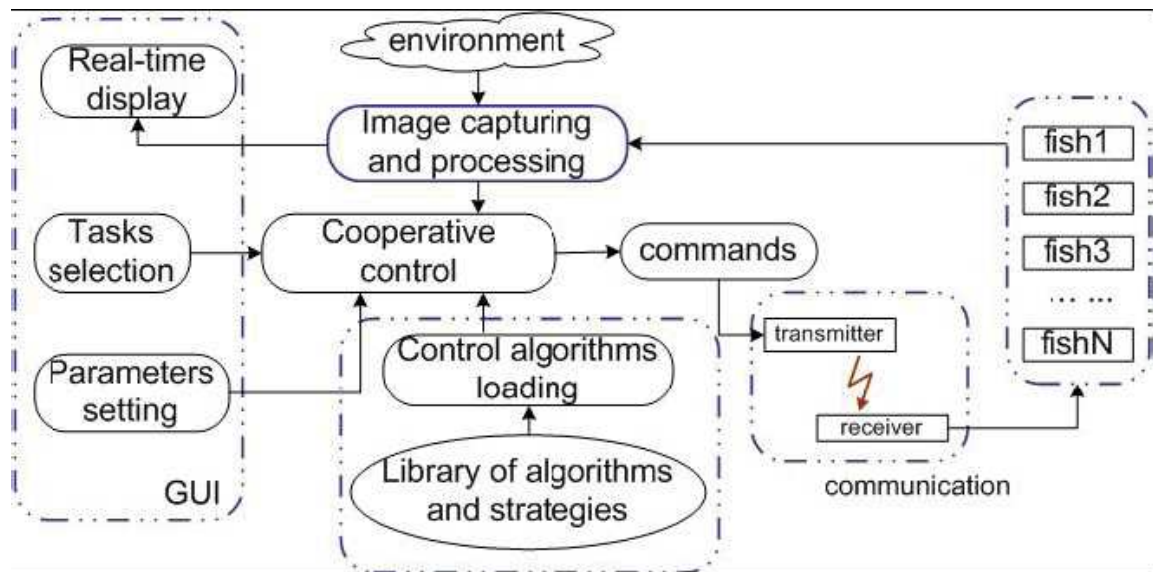

Fig. 5. Architecture of software platform

The information about fish and their surroundings are captured by an overhead camera and after being effectively processed, they are sent to the decision making and control subsystem as inputs. Then, based on input signals and specific control strategies for different tasks, the decision making subsystem produces corresponding control commands and transmits them to every robotic fish through the wireless communication subsystem. Since a global vision is adopted, MRFS should be basically categorized into centralized control system and so global planning and optimization can be obtained as a result. Based on the hardware architecture, we also develop a task- oriented software platform, on which we can implement various functions associated with cooperative task, such as task selection, environmental parameters setting, real-time display, image processing, control algorithm loading and commands executing. Fig 5 shows the schematic diagram of the software system architecture. It consists of GUI (Graphics User Interface), image processing module, algorithm module, communication module and fish module. Through GUI, users can choose different tasks, set 
parameters of environment (goals, obstacles, etc.). In image processing module, a global image information is captured and processed. After that, useful information is abstracted and used for making decision. Algorithm module contains the algorithms and strategies, determining how the fished cooperate with each other. Communication module transmits every control command to the fish module which are the actuators of MRFS.

\subsection{Hierarchical Control Algorithm for Cooperative Application}

In this subsection, we propose a hierarchical control algorithm for cooperative application on MRFS. A four-level hierarchical architecture is developed: The first level is task planner level. In this level, the required task is decomposed into different roles. During the decomposition, it should be guaranteed that these roles are necessary and sufficient for achieving the task. After producing different roles we should select the most qualified candidate of robotic fish for each role according to some proper rules. Aiming at requirements of different tasks, we introduce both static and dynamic role assignments mechanism. In static assignments, once roles are determined at the beginning of the task, they will not change during the task; while in the dynamic mechanism, the robotic fish may exchange their roles according to the progress of the task. The third level is the action level. In this level, a sequence of actions are designed for each role. By action, we mean an intended movement of fish, such as turning, advancing, and so on. The fourth level, which is called the controller level, is the lowest one. In this controller level, we give a sufficient consideration to some unfavourable factors when control due to the speciality of fish.

- When the robotic fish swims, the interaction between it and its surrounding water will result in resonance at certain frequency. Moreover, the robotic fish can't stop immediately even if the oscillating frequency is set to zero. Hydrodynamic forces and the fish's inertia will make it drift a short distance along its advancing direction.

- In our design, the robotic fish's orientation is controlled by modulating the first two joints' deflection $(\varphi 1, \varphi 2)$. However, it is quite difficult to adjust the deflection accurately, because the drag force produced by surrounding water is an unstructured disturbance and we can't get its precise model.

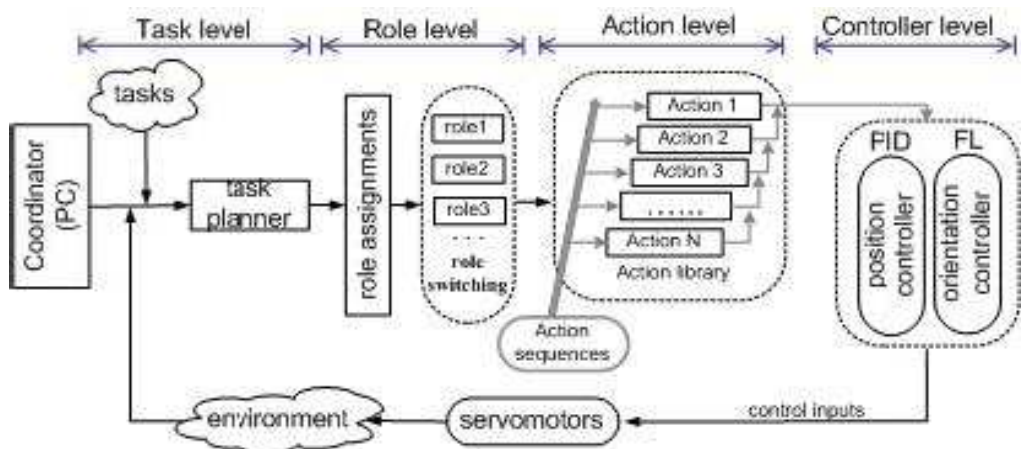

Fig. 6. The block diagram of the hierarchical architecture

Based on the above conditions, we adopt a PID controller for piecewise speed control and a 
fuzzy logic controller for orientation control, which are presented particularly in our previous work [3]. Figure 6 illustrates the block diagram of cooperative control architecture consisting of four levels.

This section concentrates on the multi-robot fish cooperation problem. It describes the design and implementation of MRFS. It has been tested that MRFS provides a useful and effective platform to design and achieve cooperative tasks for multiple robot fish. Fig.7 shows the tasks designed on the basis of MRFS:

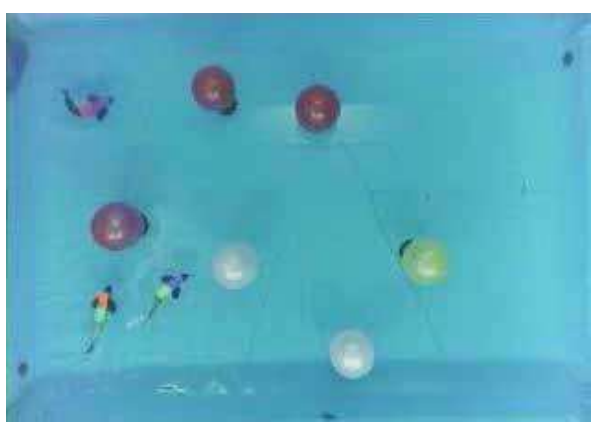

(a) Blasting collaboration

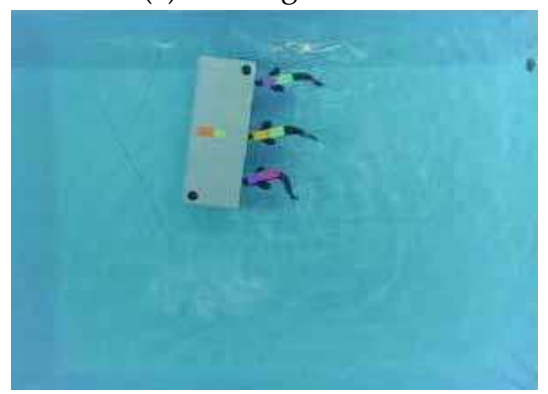

(c)Pushin Box

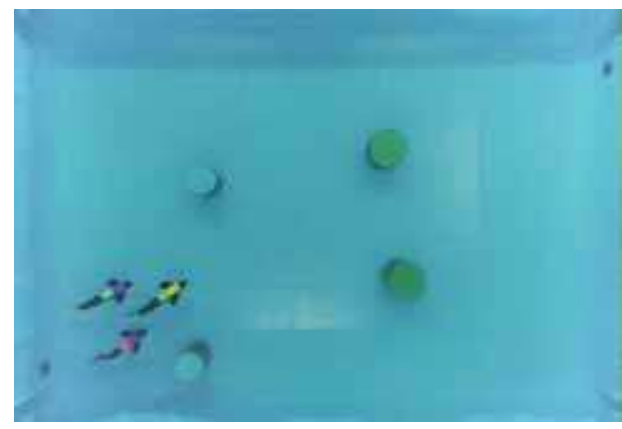

(b)Muti-fish Formation

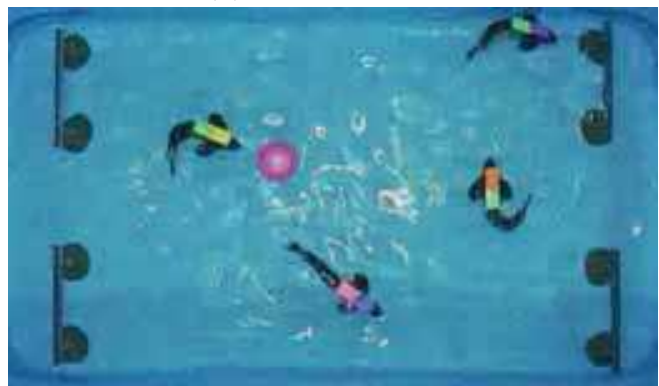

(d)Robotic Water Polo

Fig. 7. The cooperating tasks on MRFS

\section{The Collision Avoidance of Robotic Fish in RWP}

As we know, robot navigation is concerned with driving a robot to the destination along a collision free path to perform a given task. In general, the navigation methods can be classified into two categories: global navigation, which is based on a prior known environment information; reactive navigation, which is based on real-time sensory information. Global navigation methods include roadmap approach, cell decomposition approach, artificial potential field approach, electrostatic potential field and the magnetic field method, etc. These methods can be understood well from the theoretical aspect, but the computational cost is expensive, so they are not applicable in dynamic environments. In reactive navigation, the robot makes decision on the real time sensory information. The low computational cost allows this approach suitable to be used in unknown or dynamic 
environment. Examples of this approach include: Potential Fields [9], Vector Field Histogram [10], behavior-based method [11], fuzzy logic [12] and the Nearness Diagram Navigation [13]. Reactive navigation approach is effective in many dynamic scenarios, but most research on this method considers the robot as a point with holonomic properties. For the robotic fish we used in RWP, due to the particular propulsive mechanism, the translational velocity and the rotational velocity are not independent but coupled with each other. In addition, the robotic fish cannot move reversely or stop immediately in the water environment due to the inertial drift. Because of the particular kinematic characteristics of robotic fish and the complexity of the underwater environment, few navigation methods in the literature can be applied to robotic fish navigation directly. New navigation methods are required to be exploited for the robotic fish.

In this section, we propose a new reactive collision avoidance method for robotic fish navigation in the environment of RWP. Considering the nonholonomic properties and the inherent kinematic constraints of the robotic fish, limit cycle approach is proposed, with which the robotic fish can avoid one another smoothly and efficiently. Experiments performed by three robotic fish demonstrate the effectiveness of the proposed method.

The section is organized as follows. In subsection 2.1, the simplified propulsive model of the robotic fish is presented. In subsection 2.2, we describe our collision avoidance method in detail. Experimental results are given in subsection 2.3. subsection 2.4 concludes this section.

\subsection{Simplified Propulsive Model of The Robotic Fish}

Our designed robotic fish takes carangiform movement. Barrett et al. has presented a relative swimming model for RoboTuna (carangiform) in [6], and the undulatory motion is assumed to take the form of a propulsive travelling wave which is described by:

$$
\operatorname{ybody}(x ; t)=\left[\left(\mathrm{c} 1 \mathrm{x}+\mathrm{c} 2 \mathrm{x}^{2}\right)\right][\sin (k x+w t)] \text { : }
$$

In (1), ybody is the transverse displacement of the fish body, $x$ the displacement along the main axis, $k$ the body wave number $(k=2 \pi / \lambda \square), \lambda$ the body wave length, $c 1$ the linear wave amplitude envelope, $c 2$ the quadratic wave amplitude envelope, and $\omega$ the body wave frequency $(\omega=2 \pi f)$.

For simplification, we consider the discrete form of travelling wave (1), which is described by:

$$
\operatorname{ybody}(x ; i)=\left[\left(\mathrm{c} 1 \mathrm{x}+\mathrm{c} 2 \mathrm{x}^{2}\right)\right]\left[\sin \left(\mathrm{kx} \pm \frac{2 \pi}{\mathrm{M}} \mathrm{i}\right)\right]
$$

where $i$ denotes the index of the sequences, $M$ is the body wave resolution, representing the discrete degree of the overall travelling wave.

\subsection{Coordinated Collision Avoidance}

(a) Kinematic Constraints of The Robotic Fish

Due to the particular propulsive mechanism, the translational velocity $v$ and the rotational velocity of the robotic fish are not independent, but coupled with each other. Typical coupling relations of them under different oscillatory frequencies of the tail are shown in Fig. 8. We use the following equation to describe the relations: 


$$
\mathrm{w}=F(v, \text { requency })
$$

Equation (3) is called inherent constraints of the robotic fish. Any control input pair of $(v \omega)$ shall satisfy the inherent constraints.

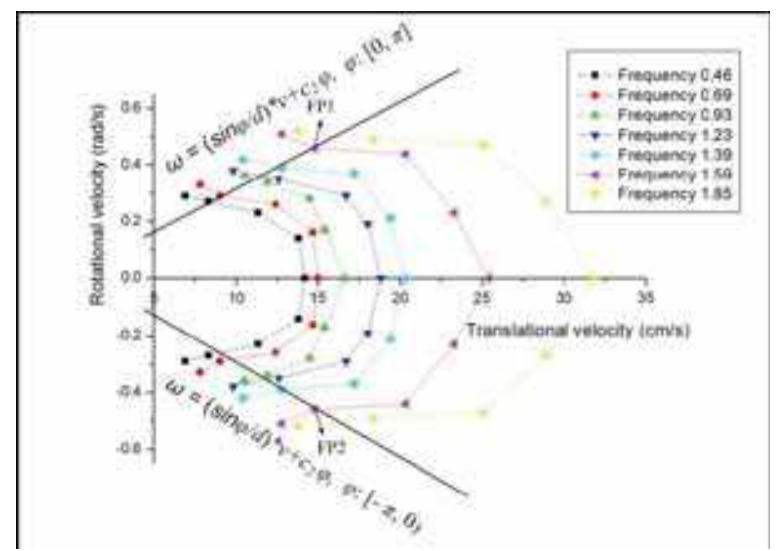

Fig. 8. Relations between the translational velocity and the rotational velocity under different oscillatory frequencies

(b) Limit Cycle Approach for Collision Avoidance

Next we discuss how collisions among multiple robotic fish are avoided. For simplification, The shape of the fish is described as an ellipse in the two dimensional plane. First we give one important lemma.Poincar'e-Bendixson Theory:

If $D$ is an annulus-shape bounded absorbing region, $D \subset R^{2}$, and contains no equilibria, then $D$ contains at least one periodic orbit. (A bounded region $D$ on the phase plane is absorbing if no trajectories leave it.)

(c) Limit Cycle Approach

Considering the following nonlinear system in the fish frame:

$$
\begin{aligned}
& \dot{\mathrm{x}}=\lambda\left(\mu \mathrm{y}+\gamma \mathrm{x}\left(\mathrm{r}^{2}-\mathrm{x}^{2}-\mu^{2} \mathrm{y}^{2}\right)\right) \\
& \dot{\mathrm{y}}=\lambda\left(-\frac{1}{\mu} \mathrm{x}+\gamma \mathrm{y}\left(\mathrm{r}^{2}-\mathrm{x}^{2}-\mu^{2} \mathrm{y}^{2}\right)\right)
\end{aligned}
$$

where $\gamma \lambda \mu r$ are positive parameters. In order to prove the ellipse $\mathrm{x}^{2}+\mu^{2} \mathrm{y}^{2}=\mathrm{r}^{2}$ is the limit cycle of system (4), we use the following Lyapunov function:

$\mathrm{v}(\mathrm{x} ; \mathrm{y})=\mathrm{x}^{2}+\mu^{2} \mathrm{y}^{2}$ :

such that:

$$
\begin{aligned}
& \mathrm{V}(\mathrm{x}, \mathrm{y})=2 \lambda \gamma\left(\mathrm{r}^{2}-\mathrm{x}^{2}-\mu^{2} \mathrm{y}^{2}\right)\left(\mathrm{x}^{2}+\mu^{2} \mathrm{y}^{2}\right) \\
& =2 \lambda \gamma\left(\mathrm{r}^{2}-\mathrm{V}(\mathrm{x}, \mathrm{y}) \mathrm{V}(\mathrm{x}, \mathrm{y})\right.
\end{aligned}
$$

We can see $\dot{\mathrm{V}}(\mathrm{x}, \mathrm{y})<0 \quad$ when $\mathrm{V}(\mathrm{x}, \mathrm{y})>\mathrm{r}^{2}$, while $\dot{\mathrm{V}}(\mathrm{x}, \mathrm{y})>0 \quad$ when $_{\mathrm{V}}(\mathrm{x}, \mathrm{y})<\mathrm{r}^{2}$. This 
shows the following annulus-shape region

$$
\mathrm{B}=\left\{\mathrm{a} 1 \leq V(x, y) \leq \mathrm{a} 2, \mid 0<\mathrm{a} 1<\mathrm{r}^{2}, \mathrm{a} 2>\mathrm{r}^{2}\right\}
$$

is absorbing. It is also bounded and free of equilibrium points, since the unique equilibrium point is $(0 ; 0)$. According to Poincar'e-Bendixson Theory, $B$ contains at least one periodic orbit. Since this argument is adaptive for any $0<a 1<r^{2}$ and $a 2>r^{2}$, when $\square \mathrm{a} 1, \square \mathrm{a} 2$ get close to $r^{2}$, region $B$ shrinks to the ellipse $V(x, y)=r^{2}$. Thus we get the limit cycle in the fish frame (shown in Fig. 9):

$$
V(x, y)=r^{2} \Rightarrow x^{2}+\mu^{2} y^{2}=r^{2}
$$

The convergence speed of $(x, y)$ toward the limit cycle can be tuned by the constant $\gamma$.Fig. 9 (a) shows the fast convergence condition with $\gamma=0.001$, and Fig. 9 (b) shows the slow convergence condition with $\gamma=0.0001$. From Fig. 9 we can see the trajectory from any point $(x, y)$ moves toward and converges to the limit cycle clockwise when close. The counterclockwise condition can be derived by the following system (shown in Fig. 10):

$$
\begin{aligned}
& \dot{\mathrm{x}}=\lambda\left(-\mu \mathrm{y}+\gamma \mathrm{x}\left(\mathrm{r}^{2}-\mathrm{x}^{2}-\mu^{2} \mathrm{y}^{2}\right)\right) \\
& \dot{\mathrm{y}}=\lambda\left(\frac{1}{\mu} \mathrm{x}+\gamma \mathrm{y}\left(\mathrm{r}^{2}-\mathrm{x}^{2}-\mu^{2} \mathrm{y}^{2}\right)\right)
\end{aligned}
$$

Since the trajectory from any point $(x, y)$ inside the limit cycle moves outward the cycle (thus away the center point of the cycle), and the trajectory from any point $(x, y)$ outside the limit cycle approaches the cycle by aparting the center point distances (determined by the cycle), the limit cycle provides a method for collision avoidance among multiple robotic fish. Shown in Fig. 12, the shape of the obstacle fish body is described by an ellipse, and the safe region is defined by a concentric safe ellipse. When another fish is in the safe region, by the limit cycle approach, it will move away the obstacle fish toward the safe ellipse. Let $\theta$ denote the orientation of the fish, $(x 0, y 0)$ the center point of the fish. With the following transformation we get the expression of system (4) in the original frame:

such that:

$$
\begin{aligned}
& x=\cos \theta(x+x 0)-\sin \theta(y+y 0) \\
& y=\sin \theta(x+x 0)+\cos \theta(y+y 0)
\end{aligned}
$$

$$
\begin{aligned}
& \dot{x}=\cos \theta \dot{x}-\sin \theta \dot{y} \\
& \dot{y}=\sin \theta \dot{x}+\cos \theta \dot{y}
\end{aligned}
$$

Let $v$ denote the translational velocity of the fish in the original frame, a the direction of the motion. The kinematic model of the robotic fish is described by: 


$$
\begin{aligned}
& \dot{x}=v \cos a \\
& \dot{y}=v \sin a \\
& \frac{\dot{y}}{\dot{x}}=\tan a
\end{aligned}
$$

Substituting (11) into (12) we get:

$$
\begin{aligned}
& v=\sqrt{\dot{x}^{2}+\dot{y}^{2}} \\
& a=\arctan \frac{\dot{y}}{\dot{x}}+\theta
\end{aligned}
$$

By tuning the value of $\lambda$, we can adjust the magnitude of $v$ to get arbitrary speed values. Advantages of the limit cycle approach are listed below:

1) Since the control inputs of the robotic fish are the translational velocity $v$ and the orientation angle a instead of the rotational velocity $\omega$, we elimitate the trouble of treating the tackled coupling between $\boldsymbol{V}$ and $\omega$ in collision avoidance.

2) It is an efficient reactive collsion avoidance approach, and the fish can avoid the obstacle with the safety distances and appropriate direction without moving far away from the obstacle.

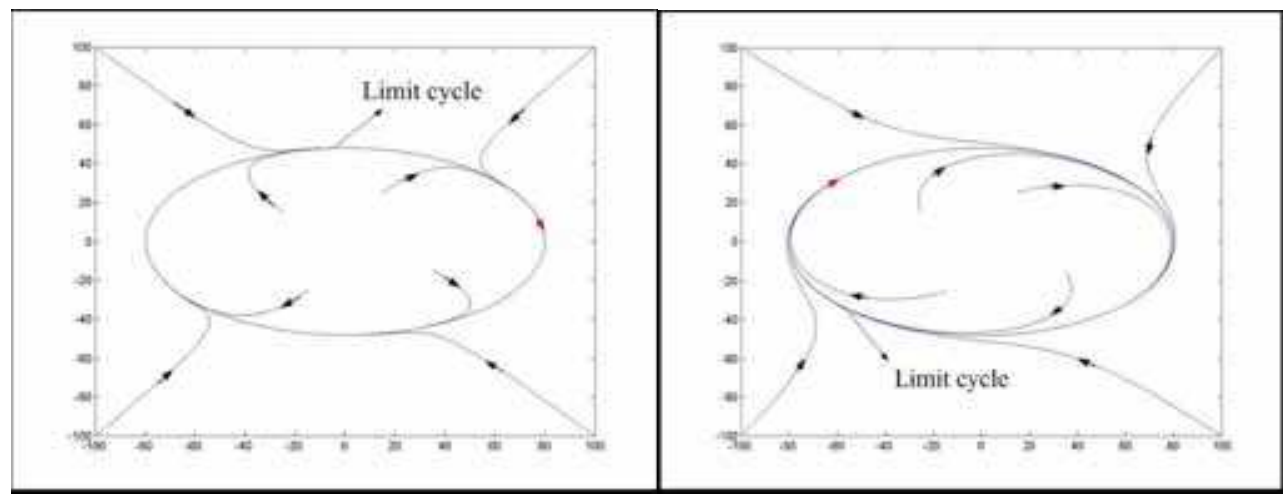

(a) Fast convergence with $\gamma=0.001$

(b) Slow convergence with $\gamma=0.0001$

Fig. 9. Phase portrait of limit cycle (clockwise) 


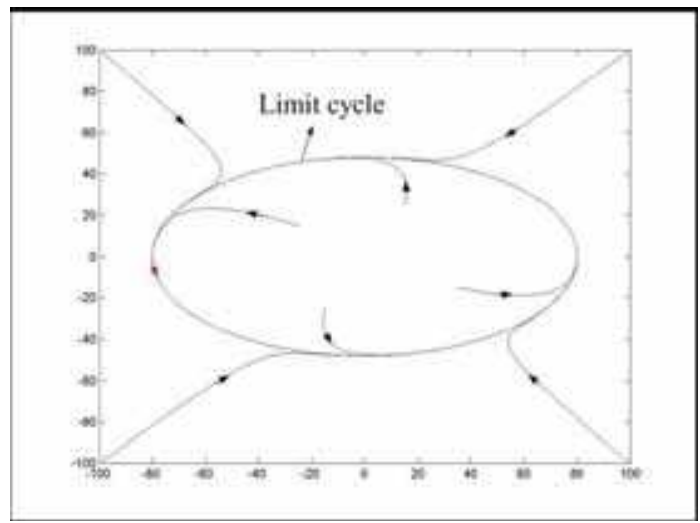

Fig. 10. Phase portrait of limit cycle (counterclockwise)

(d)Applying Limit Cycle Approach in Multiple Robotic Fish Collision Avoidance

Next we employ three robotic fish for discussion. To apply the limit cycle approach in the coordinated collision avoidance, we employ the situated-behavior. method (similar to the situated-activity paradigm (see [14])) to divide the environment into a set of exclusive and complete situations, and for each situation, a behavior is elaborately designed to solve the situation associated problem individually. The advantage of employing this method is that it is a divide and conquer. strategy, which reduces the task difficulty; in addition, the real-time behavior coordination problem need not to be taken into consideration, since the situations are complete and exclusive.

Situations Here we discuss only the situations for fish 1 . The situations for other fish are similar. We use a decision tree to define the set of situations according to the relative locations of the robotic fish. The decision tree is traversed through binary decision rules according to several criteria. As shown in Fig. 11, the inputs of the decision tree are the goal location information and sensory information from the overhead camera, including the ID information, the location and orientation information of the fish. The current

situation is identified according to the input information. The decision tree is traversed through binary decision rules according to the following four criteria.

Criterion 1: Obstruction criterion. This criterion classifies the situations into the following two categories according to whether fish 1 is obstructed by another fish:

(1) Nobs (not obstructed) situation: Fish 1 is not obstructed by any other fish;

(2) OBS (obstructed) situation: otherwise.

Criterion 2: Obstacle fish number criterion. This criterion divides OBS situation into the following two situations:

(1) Sobs (single obstructed) situation: Fish 1 is obstructed by only one fish;

(2) Tavoid (trap avoiding) situation: Fish 1 is obstructed by other two fish simultaneously (shown in Fig. 13 (d)).

Criterion 3: Dual avoiding criterion. This criterion classifies Sobs situation into the following two situations according to whether fish 1 and the other fish are obstructed by each other: 
(1) Savoid (single avoiding) situation: Fish 1 is obstructed by the other fish but not an obstacle for that fish (shown in Fig. 13 (a));

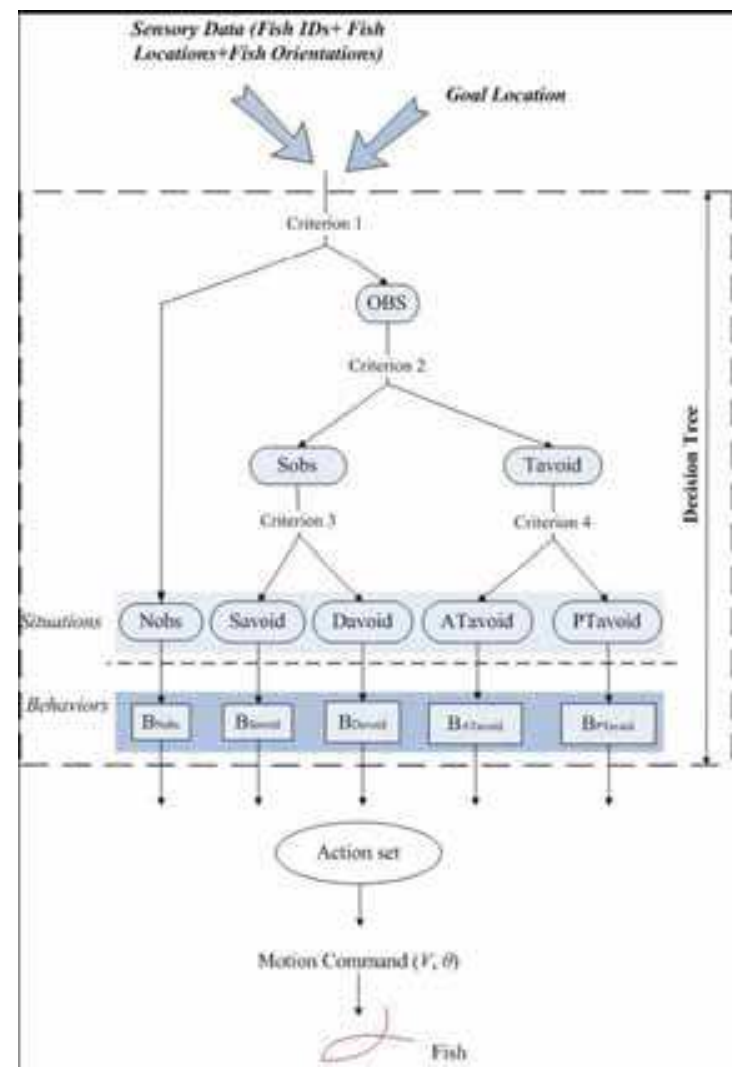

Fig. 11. Situations of coordinated collision avoidance

(2) Davoid (dual avoiding) situation: Fish 1 and the other fish are obstructed by each other (shown in Fig. 13 (b) (c)).

Criterion 4: Active avoiding criterion. According to whether fish 1 has the highest priority among fish in Tavoid situation (here the priorities of the fish are arranged according to their IDs), this criterion divides Tavoid situation into the following two situations:

(1) ATavoid (active trap avoiding) situation: Fish 1 has the highest priority;

(2) PTavoid (passive trap avoiding) situation: Otherwise.

We only care the leaf nodes of the decision tree: Nobs, Savoid, Davoid, ATavoid, PTavoid. Obtained through a binary decision tree, these five situations are exclusive and complete.

\section{Behaviors associated with the situations}

First we define a normal behavior.

normal behavior: If the goal direction is to the right of the obstacle direction, and the path of the fish to the goal location is obstructed by the safe ellipse of the obstacle fish, shown in Fig. 
12 (a), the fish moves toward the limit cycle counterclockwise; while if the goal direction is to the left of the obstacle direction, and the path of the fish to the goal location is obstructed by an obstacle fish, the fish moves toward the limit cycle clockwise, shown in Fig. 12 (b).

1) BNobs: Fish 1 approaches the goal location directly.

2) BSavoid: Fish 1 avoids the obstacle fish with normal behavior (shown in Fig. 13(a)).

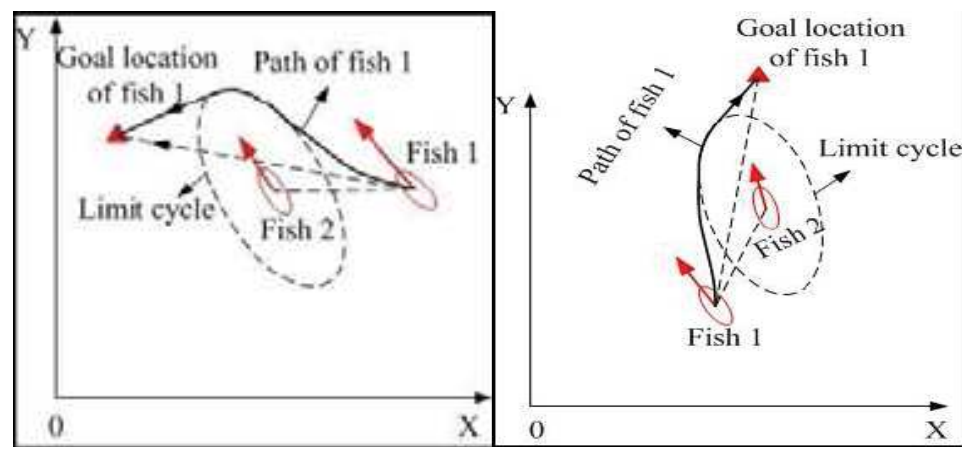

(a) Avoiding fish counterclockwise (b) Avoiding fish clockwise

Fig. 12. Normal behaviour

3) BDavoid: The two fish avoids each other with the same limit cycle direction (both clockwise, or both counterclockwise). If fish 1 is prior to the other fish, it avoids the other fish with normal behavior; otherwise it avoids the other fish with the direction opposite to normal behavior (shown in Fig. 13 (b) (c)).

4) BATavoid: In Tavoid situation, it is possible for fish 1 to get stuck in a local minima with normal behavior. For example, in Fig. 13 (d), fish 1 is avoiding fish 2 counterclockwise; however, since it is also obstructed by fish 3 , then it will avoid fish 3 clockwise. Thus fish 1 will get stuck between fish 2 and fish 3 . BATavoid is to avoid this situation. Let $l$ denote the line from fish 1 to Goal of fish 1, and fish $i(i 2(2 ; 3))$ represent the fish with the shorter distance to line $l$. Fish 1 avoids fish $i$ with direction opposite to normal behavior, until the local trap situation is eliminated (shown in Fig. 13 (d)).

BPTavoid: Fish 1 avoids the fish in ATavoid situation with the same limit cycle direction to overcome confiicts.

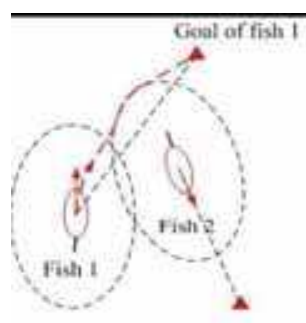

Goal of fish 2

(a) Savoid situation example

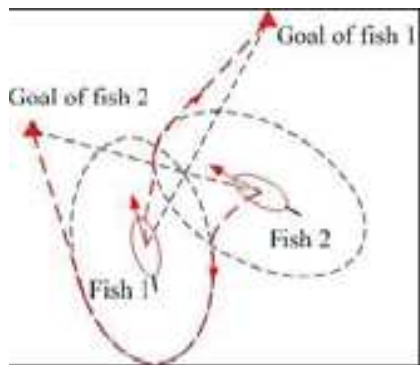

(b) Davoid situation example 1 


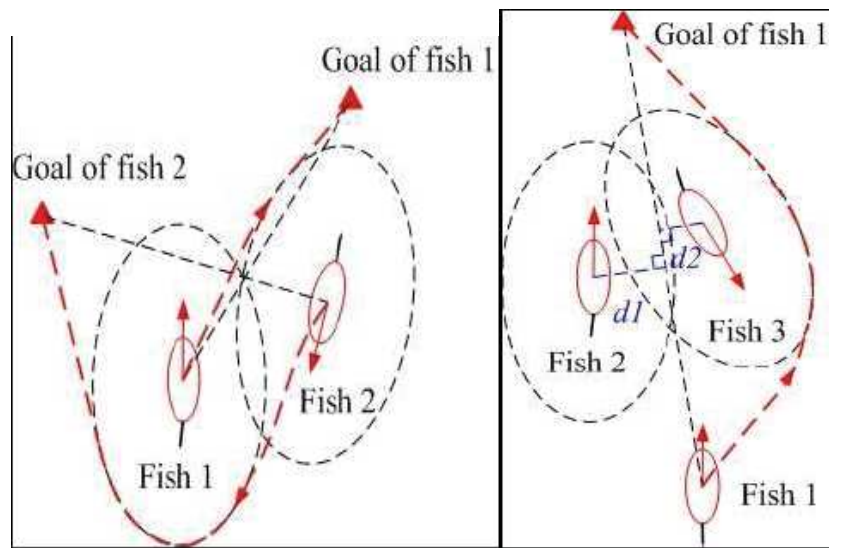

(c) Davoid situation example 2

(d) ATavoid situation example

Fig. 13. Situations and associated behavior design

\subsection{Conclusion for Collision Avoidance}

We have presented a novel collision avoidance method for multiple robotic fish Considering the inherent kinematic constraints of the robotic fish, we employ limit cycle approach for coordinated collision avoidance among the robotic fish. This approach allows the robotic fish to avoid one another smoothly and efficiently, and also eliminates the local minima problem. Experimental results demonstrate the effectiveness of the proposed method.

\section{Situation Based Action Selection and Design for Pushing Ball in RWP}

In this section, we will present the situation-base action selection mechanism to achieve ball pushing in RWP. It seems that pushing ball is quite simple, one robotic fish may be enough to finish it, in fact, the robotic fish's head is only $40 \mathrm{~mm}$ wide and the ball drifts with the fluctuant water, it is not easy for one robotic fish to touch the ball exactly in the expected point and push it to the direction of the gate. Moreover, because of its inertia and hydrodynamic forces, the robotic fish can't stop immediately even if the oscillating frequency is set to zero. It will drift a short distance along its current direction and thus, overshot occurs. When this happens, the fish must turn back and re-adjust its relative position with respect to the ball, which will take a long time. In addition,when the fish swims back and adjusts its attitudes, it will inevitably disturb the surrounding water. As a result, the ball may float away before the fish approaches it, which will increase difficulty to the game.

Based on the above consideration, multiple fish are used to push the ball to goal cooperatively. For example, we can allow two or three to push the ball in different points, using composition of their forces to move the ball towards the gate. Or if a fish overshoot 
the ball, it can hit the ball by its tail and make the ball fioat to a favorable position for another teammate.

\subsection{Working Region, Situations and Rules for the Game}

To describe the situations for the game, we first introduce three working regions for the robotic fish. As shown in Fig. 14, $l$ is a line connecting the center of the ball to the goal. Let us draw a circle at the center of the ball with a radius $r$ and then partition it into four vectors. There obtain five working regions: Pushing Region, Left Assistant Pushing Region, Right Assistant Pushing Region and Overshot Region. Other regions out of the circle is called Buffer Region.

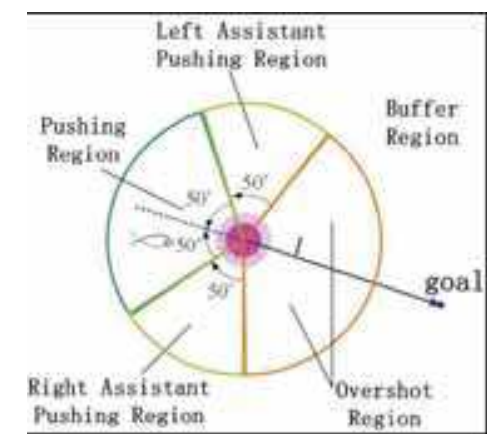

Fig. 14. Illustration of the region division

$>\quad$ the Pushing Region (PR): this is an effective working region, since in this region the fish-like robot can push the ball to the direction of the goal and so the pushing action is effective.

$>\quad$ the Left Assistant Pushing Region (LAPR): this region is a semi-effective working region. The fish in this region can not directly push the ball to the desired direction, but it can help a fish in PR prevent the ball floating away.

$>$ the Right Assistant Pushing Region (RAPR): similar to LAPR, this is also a semi-effective working region

$>\quad$ the Overshot Region (OR): this region is a forbidden working region. When the fish swims into this region, it will be located between the ball and the goal. In this region, the fish are forbidden to touch the ball because it will push the ball to the opposite direction to the destination. In this case, the fish should first swim around the ball and enter a effective working region and then select suitable actions.

$>\quad$ the Buffer Region (BR): In this region, the fish is a little bit far from the ball, in will try to swim to an effective region (PR, LAPR or RAPR) first.

We use multiple fish in the RWP in order to enhance the efficiency of the game. However, when the number of the fish increase, more problems may occur. Because the space is limited, these fish may collide with each other. In order to deal with such circumstance, the collision avoiding strategies are designed, which will debase the performance of cooperation.Moreover, when multiple fish swim in the same place, the surrounding water 
will be disturbed violently. The ball will float everywhere and become more difficult to track and push. Additionally, waves produces by fish and ball may cause uncertainty and inaccuracy to the image information captured by the overhead camera..Considering the characteristics of the fish's dynamics and the speciality of the hydro-environments, we propose the following rules for this game.

Rules 1: as shown in Fig. 15 (a), we divide the space (pond) into three parts in Y coordinates: Part A, Part B and Part C, representing the respective region each fish take charge of. We use such kind of region-responsibility strategy with main intent to avoid collisions between these fish. For each fish, if the ball is not in the region that it is responsible for. It will still swim restrictively within its region and cannot invade other regions to disturb its teammates. This rule is inspired by the human soccer match in which different roles take charge of different regions.

Rules 2: we prefer slowness than overshoot when controlling the fish. We intent to slow down the fish to some extent, especially when it approaches the pushing point. In addition, the distance from the pushing-point to the center of the ball is defined to be a value larger than theoretical value.

Rules 3: as we mentioned, the fish can't stop immediately even you send stop command. It will drift a short distance out of control. So, in practice, even when the fish is idle, we will let the fish wander or move very slowly instead of using stop command to halt it.

Obviously, based on the above rules and according to the number of fish entering the Pushing Region, we can define four primary situations for the task.

Situation 0 (S0): no fish is in the Pushing Region. In this situation, the game can not be implemented immediately. The fish should first swim entering the effective working region. Situation 1 (S1): there is only one fish-like robot in the Pushing Region. In order to effectively push the ball, the fish should touch the ball exactly to the direction of the goal. In this situation, according to the number of fish in the Assistant Pushing Regions we can define three sub-Situations:

$>\quad$ S1---APR0: there is no fish in the Assistant Pushing Regions

$>$ S1----LAPR1: there is one fish in the Left Assistant Pushing Regions, so this fish will assist the main pusher to transport the ball.

$>$ S1----RAPR1: there is one fish in the Right Assistant Pushing Regions.

$>$ S1----APR2: two fish are in the Assistant Pushing Regions, one is left and one is right.

Situation 2 (S2): there two fish are in the pushing region. At this circumstance, these two fish can push the ball cooperatively. They push the ball from different points and let the combination of their force move the ball to the destination. Similarly, we can define two sub-Situations:

$>$ S2----APR0: there is no fish in the Assistant Pushing Regions

$>$ S2----LAPR1: there is one fish in the Left Assistant Pushing Regions, so this fish act as an assistant in the game.

$>\quad$ S2---RAPR1: there is one fish in the Right Assistant Pushing Regions.

Situation 3 (S3): all the fish are in the pushing region. In this situation, these fish can have a work division. Some are responsible for pushing ball, others can assistant the pusher by preventing the ball from floating away with the fluctuant water. 
Different situations will result in different task difficulty. As we mentioned above, when there is only one fish pushing the ball, it may easily miss the ball or push it to the wrong direction. While, as the pushers increase, the game will be easier due to cooperation and redundancy. So, in order to push the ball in a more stable and precise way, multiple fish are expected to help each other and work in a cooperative way, such as in S2 and S3.

\subsection{Situated Actions Design}

In contrast to controlling some ground mobile robots, the robotic fish's attitudes are more difficult to modulate, because it is very difficult to establish any precise dynamics models for the robotic fish. Hence, we designed the fish's primitive actions based on geometric approach:

Action 0: This action is designed for the fish to swim from a non-pushing region to the effective working region. Basically, this is a simple Point----To----Point(PTP) control.

Action 1: As depicted in Fig. 15 (b), the first action for the fish is to swim approaching the ball and hit the ball exactly along the direction from the ball to the gate. where $\left(x_{F}, y_{F}\right.$, $\alpha \square)$ denotes the pose of the fish, $\left(x_{D} ; y_{D}\right)$ and $\left(x_{C} ; y_{C}\right)$ stand for the center of the ball and the position of the gate, $\beta$ is the expected direction from the ball to the gate, $l_{1}$ indicates the expected moving direction of the ball. Considering the fish's bodylength and its inertia, We choose a point $G\left(x_{G} ; y_{G}\right)$ which located at the extended line of $l_{1}$ as the pushing-point. $l_{2}$ is the section connecting the fish to $G$, and $l_{3}$ represents the perpendicular of $l_{1}$ which pass through point $G$.

As illustrated in Fig. 15 (b), if the pushing-point $G$ locates between the fish and the ball, that's $\left(x_{D}-x_{F}\right) \times\left(x_{C}-x_{D}\right)>0$, we first define the perpendicular bisector $l_{4}$ for section $l_{2}$. Then using $r$ as radius, we make a circle $C$ which is tangental to $l_{1}$ at point $G$. If the circle intersects $l_{4}$ at one point, we chose this point as a temporary goal for the fish, or if they are two intersections, we chose the point with small $x$-coordinates, namely $T$ as the temporary goal. While, if the fish is far away from the ball and there is no intersection between $C$ and $l_{1}, G$ will be the temporary goal point for the fish. As the fish moves, a series of temporary goals will be obtained, which will lead the fish swim gradually to the pushing-point. 


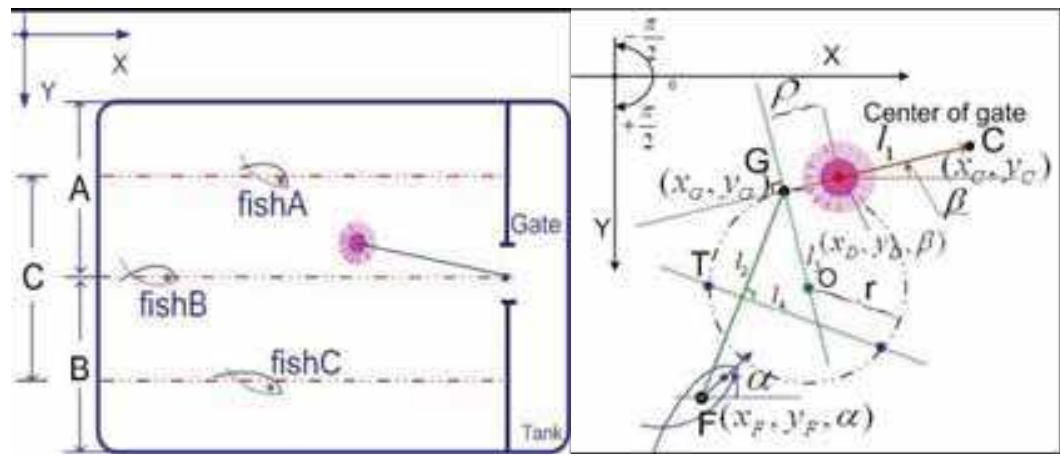

Fig. 15. (a)The experiment setting and Region division along the exact direction pointing the gate

(b)The action of pushing ball

After simple geometrical analysis, The positions of intersection points can be calculated by the following equation(14):

equation:

$$
\begin{aligned}
& \left(x-x_{D}-\rho \cos \beta-r \sin a\right)^{2}+\left(y-y_{D}-\rho \sin \beta+r \cos a\right)^{2}=r^{2} \\
& y-\frac{1}{2}\left(y_{G}+y_{F}\right)=\frac{x_{F}-x_{G}}{y_{G}-y_{F}}\left(x-\frac{1}{2}\left(x_{G}+x_{F}\right)\right)
\end{aligned}
$$

Action 2: Although when determining the pushing-point, we give sufficient consideration for the dynamics of the fish and the difficulty when controlling it, we still can't guarantee the fish will reach its destination in the expected attitudes, especially its orientation. Once it gets to the pushing-point with large orientation error, it may possible miss the ball. In this case, we design the following action which allow the fish to push the ball by swing.

As shown in Fig. 16 (a), if the fish approaches the pushing-point (in a small neighbor region) and its orientation satisfies the following condition, it will take a sharp turn to the direction of the ball.

$$
\left\{\begin{array}{l}
\left(x_{F}, y_{F}\right) \in\left\{\left\|\left(x_{G}, y_{G}\right)-\left(x_{F}, y_{F}\right)\right\| \leq \delta\right\} \\
\alpha \in\{|\alpha-\beta| \geq \xi\}
\end{array}\right.
$$

where $\delta$ and $\xi$ are the bounds for position error and orientation error, which are determined empirically experiments. In our experiment, we choose $\delta=5 \mathrm{~cm}$ and $\xi=\frac{\pi}{15}$ Action 3: This action is an assistant action, which is implemented in LAPR or RAPR. In particular, this action takes full advantage of the agility of fish's tail. Fig. 16 (b) indicates the fish pat the ball by its tail. 
Action 4: Action 4 will be implemented when the ball fioats very close to the gate and to the edge of the pond, and the fish is in LAPR or RAPR. This action allows the fish to move the ball slowly towards the goal by oscillating. As shown in Fig. 16 (c), in this action, we design a point outside the pond as a virtual pushing--- point. Thus the fish will always try to swim to that virtual destination, although it can never reach it. During moving (or struggling), its body, especially posterior body, oscillates continually, which disturbs the water and makes the ball fioat towards the gate.

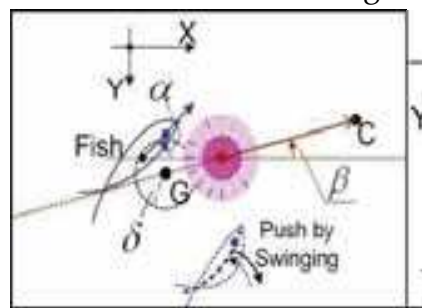

(a)

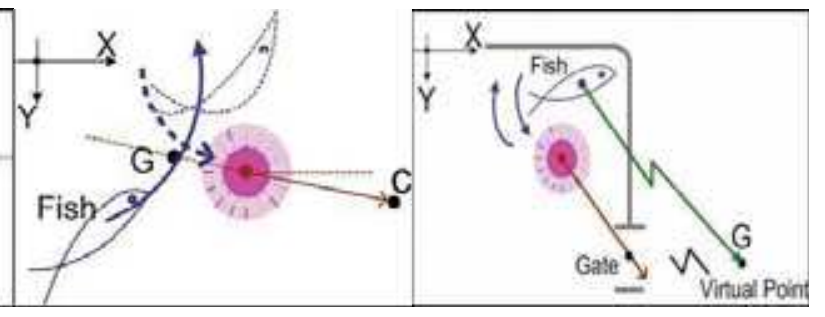

(b)

(c)

Fig. 16. The fish pushes ball by (a) shaking(throwing) head, (b)pushing the ball by tail and (c) swimming towards a virtual pushing point respectively

Next, we will investigate how the fish work cooperatively to push ball based on the above four basic actions. Three behaviors for cooperation are designed, two for two fish and one for three.

Action 5: This action is designed for S2. As shown in Fig. 17 (a), for a given pose of the ball, instead of

choosing one pushing-point, two goal points (PushPointL and PushPointR) are defined which locate at different sides of pushing-point with a defiection $\varphi$. Two fish swim towards different pushing points, and the combination of their pushing force will make the ball fioat towards the gate.

Action 6: When in S3 we adopt the following cooperative action, as shown in Fig. 17 (b). That's three fish surround the ball and sent it into the gate. At this circumstance, FishC push the ball exactly to the direction of the goal, while FishL and FishR hit the ball from PushPointL and PushPointR respectively.

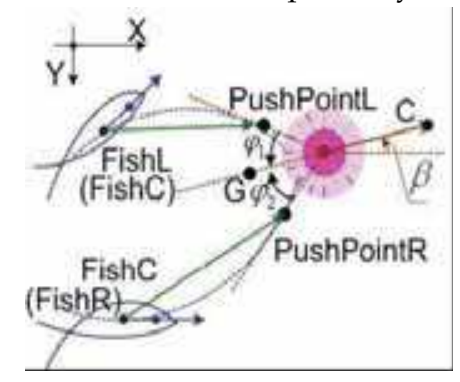

Fig.17. (a)Cooperative action for two fish.

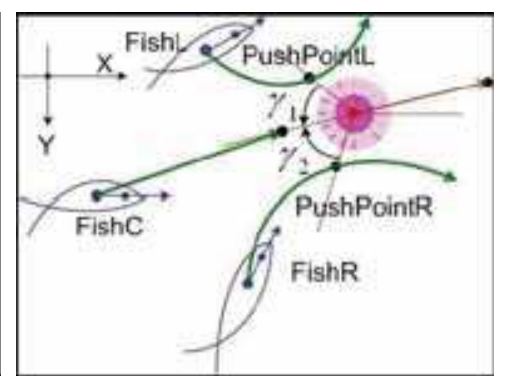

(b) Cooperative action for three fish

Table 1 sums up the corresponding strategies for each fish individual when in different situations: 


\begin{tabular}{|c|c|c|c|}
\hline \multirow[t]{2}{*}{ Situation } & \multicolumn{3}{|c|}{ Multi-robot fish } \\
\hline & FishL & FishC & FishR \\
\hline SO & Action 0 & Action 0 & Action 0 \\
\hline \multirow[t]{3}{*}{ S1-APR0 } & Action 1,2 & Action 0 & Action 0 \\
\hline & Action 0 & Action 1,2 & Action 0 \\
\hline & Action 0 & Action 0 & Action 1,2 \\
\hline \multirow[t]{2}{*}{ S1-LAPR1 } & Action 3,4 & $\begin{array}{l}\text { Action } \\
1,2\end{array}$ & Action 0 \\
\hline & Action 0 & $\begin{array}{l}\text { Action } \\
3,4\end{array}$ & Action 1,2 \\
\hline \multirow[t]{2}{*}{ S1-RAPR1 } & Action 0 & $\begin{array}{l}\text { Action } \\
1,2\end{array}$ & Action3,4 \\
\hline & Action 1,2 & $\begin{array}{l}\text { Action } \\
3,4\end{array}$ & Action 0 \\
\hline S1-APR2 & Action3,4 & $\begin{array}{l}\text { Action1 } \\
, 2\end{array}$ & Action 3,4 \\
\hline \multirow[t]{2}{*}{ S2-APR0 } & Action 0 & $\begin{array}{l}\text { Action } \\
1,2\end{array}$ & Action 1,2 \\
\hline & Action 1,2 & Action1,2 & Action 0 \\
\hline S2-LAPR1 & Action 3,4 & Action 5 & Action 5 \\
\hline S2-RAPR1 & Action 5 & Action 5 & Action 3,4 \\
\hline S3 & Action 6 & Action 6 & Action 6 \\
\hline
\end{tabular}

Table 1. Situation Based Action Selection

Fig. 18 shows the scenarios in one of the experiments. The experiment is quite successful and has high efficiency, since during the pushing, FishL and FishR cooperative very well and neither of them overshoots the ball. Moreover, the ball is relatively stable and there is little disturbance when it floats. In this sense, the hydro-environment is more complicated than general ground environment.

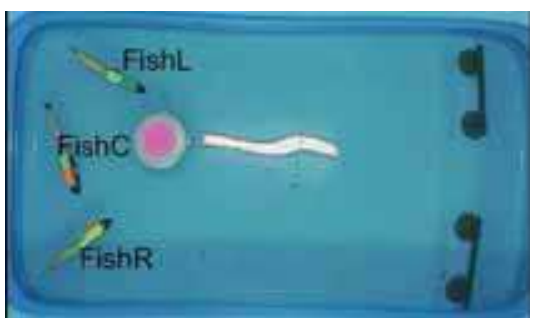

(a) $0 \mathrm{~s}$ after started

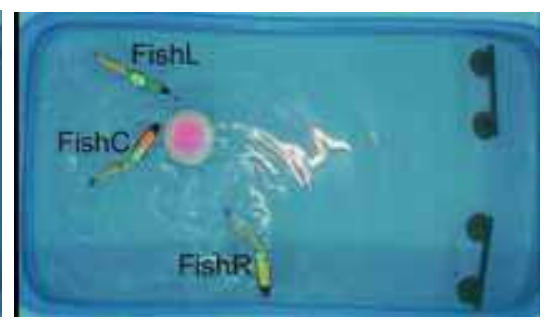

(a) $6 \mathrm{~s}$ after started 


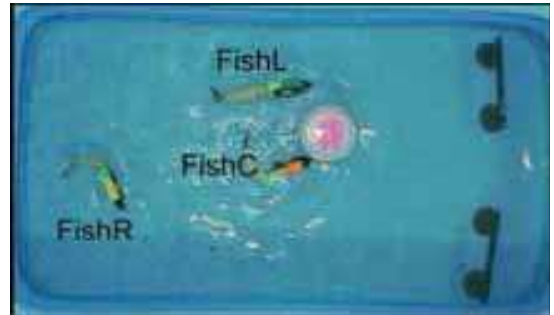

(a) $18 \mathrm{~s}$ after started

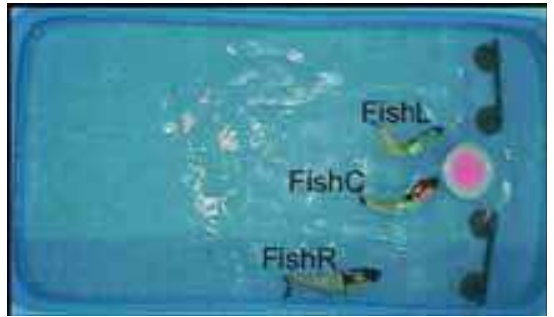

(a) $24 \mathrm{~s}$ after started

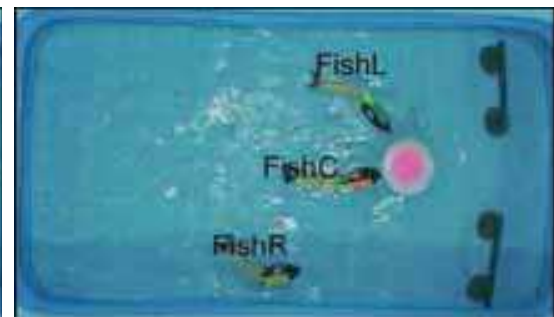

(a) $23 \mathrm{~s}$ after started

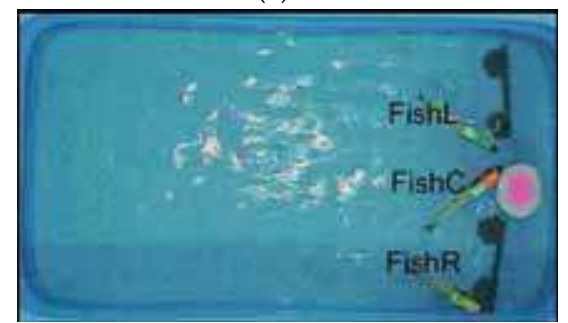

(a) $27 \mathrm{~s}$ after started

Fig. 18. Fragment of the experiment, Sequences of overhead images, order is left to right and top to down. (a) The initial state of the experiment. (b) FishL is in the LAPR, FishC in PR attempts to push the ball directly and FishR tries to approach the ball but turns when swims out of its charged region. (c) FishL takes Action 2 to pat the ball and at the same time FishC takes Action 1 to hit the ball. FishR still tries to approach the object. (d) Both FishL and FishC take Action 5 to push the ball cooperatively. FishR takes Action 0. (e) FishL takes Action 3, FishC takes Action 1 and FishR still takes Action 0. (f) FishL takes Action 3, FishC takes Action 1 and FishR takes Action 4

\section{Conclusions and Foregrounds}

We focus this chapter on the RWP and the underwater robot cooperation involved in the game, two useful methods are introduced in this chapter for the game. These two methods have been certified by the experiments.

Mankind invented the robot in the 20th century and realized the use of robots initially. In the 21st century, humans will coexist with robots. Robot Football is the ideal model to research the future sociality.

A very wide range of fields are involved in the RWP, including machinery and electronics, robotics and sensor information fusion, intelligent control, communications, computer vision, computer graphics, artificial intelligence and so on. What is more significant is that the RWP makes the research and education combined. The game combined ingeniously with the academic research will furthermore inspire the young students interesting strongly. It will train young students to have rigorous scientific attitude and good skills through the competition of RWP.

Though RWP has only just started, the significance of industry research and the huge potential has already been demonstrated: 
(1) It provides a high-tech display of the outcome of the visualization window to promote scientific technology and social production and living closer integration. Soccer is not only one of the world's most popular sports, but also a huge industry. In the current world, the research on robots always consciously takes the initiative to integrate into the community's economic and cultural life.

(2) The RWP provides an approach of converting research results to industry. It can be expected that with the development of the RWP, variety of new underwater robots will be developed, and the efficiency will grow rapidly. Also, with other types of robot soccer team and the technical aspects mature gradually, the related products will grow rapidly.

(3) RWP provides a standard platform to research on underwater robot collaboration. Due to the special nature of the underwater environment, many ground collaboration theory and algorithms can not be applied directly in the underwater environment, there are great difficulties in collaborative research on underwater robot. By providing a standard platform, it will attract more people to conduct underwater robot for underwater robot collaborative research.

RWP can also reflect comprehensive strength of a national information technology and automation technology and at the same time multiple underwater robots cooperation have broad application prospects in a large of engineering field, for example, the cooperation control research of multiple underwater robot fish has a broad prospect in the military, the detection of underwater resources, on the sea detecting and rescue, marine multi-sensor sampling network, and other military tasks in which the cooperation control research plays an important role.

In short, the RWP not only is the high-tech competition, but also has the property of view and admire and entertainment like the football game. Therefore, there will surely attract a large number of "fans" .Currently, there are more and more schools invited to participate in RWP. We have the reason to believe that under the efforts of all colleges and universities, RWP will be promoted in the world to be the standards multi-robot competition.

\section{References}

J. Yu, S. Wang and M. Tan, .Basic motion control of a free-swimming biomimetic robot fish, in Proc. IEEE Conf. Decision and Control, Maui, Hawaii USA, pp. 1268-1273, December 2003.

J. Yu, and L. Wang: Parameter optimization of simplified propulsive model for biomimeticrobot fish. In Proc. IEEE Int. Conf. Robotics and Automation. Barcelona, Spain (2005) 3317-3322

J. Yu, L. Wang, and M. Tan: A Framework for Biomimetic Robot Fish's Design and Its Realization. In Proc. of American Control Conference. Portland, USA (2005) 1593-1598

J. Yu, Y. Fang,W. Zhao, and L.Wang: Behavioral Design and Strategy for Cooperative Multiple Biomimetic Robot Fish System. In Proc. IEEE Int. Conf. Robotics and Biomimetics. Hongkong and Macau (2005) 472-477

Y. Fang, J. Yu, R. Fan, L. Wang, and G. Xie: Performance Optimization and CoordinatedControl of Multiple Biomimetic Robotic Fish. In Proc. IEEE Int. Conf. Robotics and Biomimetics. Hongkong and Macau (2005) 206-211 
D. Barrett, M. Triantafyllou, D. K. P. Yue, M. A. Grosenbaugh, and M. J. Wolfgang: Dragreduction in fish-like locomotion. J. Fluid Mech. Vol. 392. (1999) 183-212

D. Barrett, M. Grosenbaugh, and M. Triantafyllou, .The optimal control of a fiexible hull robotic undersea vehicle propelled by an oscillating foil,. in Proc. IEEE AUV Symp. pp. 1-9. vol. 241999

M. Sfakiotakis, D. M. Lane, and J. B. C. Davies: Review of fish swimming modes for aquaticlocomotion. IEEE J. Oceanic Eng. Vol. 24. (1999) 237-252

N. C. Tsourveloudis, K. P. Valavanis and T. Hebert: Autonomous vehicle navigation utilizing electrostatic potential fields and fuzzy logic. IEEE Trans. Robotics and Automation. Vol. 17.(2001) 490-497

W. Tianmiao and Z. Bo: Time-varing potential field based perception-action. behaviors of mobile robot. In Proc. IEEE Int. Conf. Robotics and Automation. (1992)2549-2554

J. Borenstein and Y. Koren: The vector field histogram-fast obstacle avoidance for mobile robots. IEEE Trans. Robotics and Automation. Vol. 7. (1991) 278-288 A. Taliansky and N. Shimkin: Behavior-based navigation for an indoor mobile robot. In The 21st IEEE Convention of Electrical and Electronic Engineers in Israel. (2000) 281-284

T.E. Mora and E.N. Sanchez: Fuzzy logic-based real-time navigation controller for a mobilerobot. In Proc. IEEE Int. Conf. Intelligent Robots and Systems. Vol. 1. (1998) 612-617

J. Minguez and L. Montano: Nearness Diagram (ND) Navigation: Collision Avoidance inTroublesome Scenarios. IEEE Trans. Robotics and Automation. Vol. 20. (2004) 45-59

R. C. Arkin: Behavior-Based Robotics. Cambridge, MA: MIT Press (1999)

Triantafyllou, M. S., Triantafyllou,G. S.: An efficient swimming machine. Sci. Amer. 272 (1995)64-70

Terada, Y.: A trial for animatronic system including aquatic robots, J. Robot. Soc. Jpn. 18(2000)195-197

Domenici, P., Blake, R. W.: The kinematics and performance of fish fast-start swimming,J. Exper. Biol., 200 (1997)1165-1178

M. S. Triantafyllou and G. S. Triantafyllou : An efficient swimmingmachine. Sci. Amer. vol. 272 pp. 64-70, 1995

M. J. Lighthill, .Note on the swimming of slender fish,. J. Fluid Mech., vol. 9, pp. 305-317, 1960.

D. Barrett, M. Triantafyllou, D. K. P. Yue, M. A. Grosenbaugh, and M. J. Wolfgang,.Drag reduction in fish-like locomotion,. J. Fluid Mech., vol. 392, pp. 183-212, 1999.

Y. Terada,. A trial for animatronic system including aquatic robots., J. Robot. Soc. Jpn. vol. 18 pp. $195-1972000$

T. Yuuzi and I. Yamamoto An Animatronic System Including Lifelike Robotic Fish pp. 1814-1820

P. Domenici and R. W. Blake, .The kinematics and performance of fish fast-start swimming, J. Exper. Biol., vol. 200, pp. 1165C1178, 1997. 


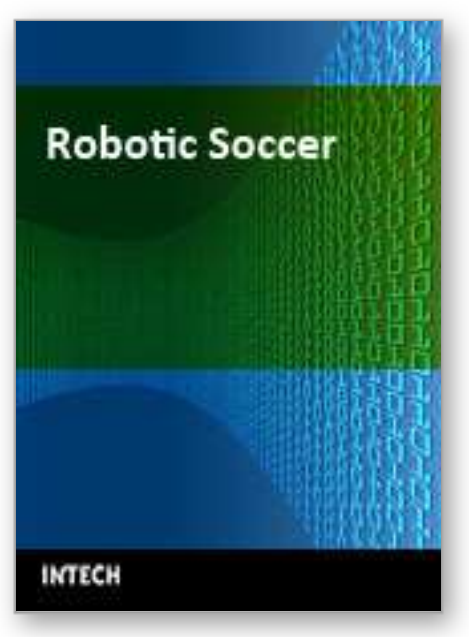

\author{
Robotic Soccer \\ Edited by Pedro Lima
}

ISBN 978-3-902613-21-9

Hard cover, 598 pages

Publisher I-Tech Education and Publishing

Published online 01, December, 2007

Published in print edition December, 2007

Many papers in the book concern advanced research on (multi-)robot subsystems, naturally motivated by the challenges posed by robot soccer, but certainly applicable to other domains: reasoning, multi-criteria decisionmaking, behavior and team coordination, cooperative perception, localization, mobility systems (namely omnidirectional wheeled motion, as well as quadruped and biped locomotion, all strongly developed within RoboCup), and even a couple of papers on a topic apparently solved before Soccer Robotics - color segmentation - but for which several new algorithms were introduced since the mid-nineties by researchers on the field, to solve dynamic illumination and fast color segmentation problems, among others. This book is certainly a small sample of the research activity on Soccer Robotics going on around the globe as you read it, but it surely covers a good deal of what has been done in the field recently, and as such it works as a valuable source for researchers interested in the involved subjects, whether they are currently "soccer roboticists" or not.

\title{
How to reference
}

In order to correctly reference this scholarly work, feel free to copy and paste the following:

Zhang Lee, Guangming Xie, Dandan Zhang and Jinyan Shao (2007). The Robotic Water Polo and Underwater Robot Cooperation Involved in the Game, Robotic Soccer, Pedro Lima (Ed.), ISBN: 978-3-902613-21-9, InTech, Available from:

http://www.intechopen.com/books/robotic_soccer/the_robotic_water_polo_and_underwater_robot_cooperation _involved_in_the_game

\section{INTECH}

open science | open minds

\author{
InTech Europe \\ University Campus STeP Ri \\ Slavka Krautzeka 83/A \\ 51000 Rijeka, Croatia \\ Phone: +385 (51) 770447 \\ Fax: +385 (51) 686166 \\ www.intechopen.com
}

\author{
InTech China \\ Unit 405, Office Block, Hotel Equatorial Shanghai \\ No.65, Yan An Road (West), Shanghai, 200040, China \\ 中国上海市延安西路65号上海国际贵都大饭店办公楼405单元 \\ Phone: +86-21-62489820 \\ Fax: +86-21-62489821
}


(C) 2007 The Author(s). Licensee IntechOpen. This chapter is distributed under the terms of the Creative Commons Attribution-NonCommercial-ShareAlike-3.0 License, which permits use, distribution and reproduction for non-commercial purposes, provided the original is properly cited and derivative works building on this content are distributed under the same license. 